\section{EFFECT OF THE DYNAMIC} REFLECTIVITY ON LASER ENERGY ABSORPTION BY ZINC: NUMERICAL TWOTEMPERATURE MODELING

\begin{abstract}
SERGEY A. LIZUNOV(1,2), VLADIMIR P. ZHUKOV(1,3), ALEXANDER V. BULGAKOV(1,2), NADEZHDA M. BULGAKOVA ${ }^{(1,2)}$
\end{abstract}

(1)HiLASE Centre, Institute of Physics CAS, Dolni Brezany, Czech Republic

(2)S.S. Kutateladze Institute of Thermophysics SB RAS, Novosibirsk, Russia

(3)Institute of Computational Technologies SB RAS, Novosibirsk, Russia

DOI : 10.17973/MMSJ.2019_12_2019101

lizunov@fzu.cz

The optical response of metal surfaces to high-power laser excitation (HPLE) is one of important topics of laser-matter interaction, which remains to be poorly understood. It is known that certain metals, which are well reflective at normal conditions, become considerably absorbing during irradiation by ultrashort high-power lasers. Reflectivity of such metals during the irradiation stage is a key parameter of the HPLE process as it determines the absorbed laser energy and, hence, the postirradiation evolution of the material. In this paper, we present a two-temperature model of femtosecond laser irradiation of zinc, which takes into account the dynamic change of surface reflectivity due to excitation of the conduction electrons. The model involves integration of the reflected part of the laser beam over the irradiation spot to enable comparisons with experimental data. The numerical scheme which combines the description of the reflectivity change with the TTM is discussed in detail.

KEYWORDS

ultrafast laser, zinc, dynamic reflectivity, electron heat conductivity, numerical modeling, two-temperature model, finite difference method

\section{INTRODUCTION}

Ultrafast laser ablation is widely used in industries and scientific research in many applications areas such as synthesis of nanoparticles [Eliezer 2004], nanostructured film deposition [Mailis 1999], surface nanostructuring [Dostavalov 2019], modification of optical properties [Vorobyev 2015], surface wetting control [Vorobyev 2009], and others. Absorption of the energy of ultrafast laser pulses is a key process as the dose of irradiation obtained by a material determines its further evolution and strongly influences its modified properties. By the date, it is known that surface reflectivity of metals can be strongly varying, depending on laser fluence applied, [Kirkwood 2009, Vorobyev 2011, Ashitkov 2016] pointing out that the highly-excited electronic subsystem in a metal absorbs laser radiation with much higher efficiency as compared to cold, slightly-excited surface. The physical reasons of this effect are not fully understood yet and even cumbersome $a b$ initio simulations are not able to describe this phenomenon [Chen 2013]. However, even a small variation in laser processing parameters can cause a heavy damage to processed material, incompatible with the goals of a particular application.

Currently, diagnostic methods do not exist, which would provide enough resolution to directly follow the complexity of the processes at temporal scales of ultrashort laser pulse action and, simultaneously at nanometer spatial scales. Measurements of reflectivity usually provide information on the integral reflected energy over the pulse action. In such circumstances, multiphysics computer simulations present an important mean for nanoscopic following of material excitation to gain insight into the spatiotemporal dynamics of optical material response. By the date, the majority of theoretical analysis and numerical simulations to understand the dynamic change of optical response of metals to ultrashort laser irradiation have been performed for gold as for one of the most studied materials (see [Ren 2011, Ashitkov 2016] and references therein). Although zinc is a corrosion-resistant metal, which is used in various technological applications where its laser processing is demanded [Klini 2008, Fang 2017], only few attempts are known on numerical studies of ultrashort laser action on this material [Shugaev 2010], which do not gain insight into dynamics of its optical properties.

In this work, we describe a model of optical response of zinc which can also be applicable to other metals. The model is based on the two-temperature model (TTM) of laser energy absorption by metal [Anisimov 1974, Wellershoff 1999, Bulgakova 2002a], which is combined with an advanced model describing the dynamics of reflectivity and absorptivity of metal under investigation. The model is extended to a $2 \mathrm{D}$ case with the purpose to integrate the reflected energy from the laser irradiation spot that is important for direct comparison with experimental data. The model allows to follow the electronlattice relaxation that enables to predict post-irradiation evolution material. The numerical method and the algorithm used for simulations are described in detail. The developed code has been verified in respect of precision of the simulations, showing a high accuracy of the modelling data.

\section{MODEL}

\subsection{Main equations}

Heating of metals by femtosecond laser pulses is usually described by the TTM, which implements the temperature of conduction electrons $T_{e}$ that differs from the lattice temperature $T_{1}$. The heat transfer in our simulations is described for onedimension, toward the material depth, that is justified by the depth of laser energy penetration (tens-hundreds of nanometers) being much smaller than the size of the irradiation spot (typical diameters of irradiated area in experiments with femtosecond lasers are in the range 10-100 $\mu \mathrm{m}$ ). Therefore, the one-dimensional equations of the TTM can be written as

$C_{e} \frac{\partial T_{e}}{\partial t}=\frac{\partial}{\partial x}\left(K_{e} \frac{\partial T_{e}}{\partial x}\right)-g\left(T_{e}-T_{l}\right)+S(x, t)$,

$C_{l} \frac{\partial T_{l}}{\partial t}=\frac{\partial}{\partial x}\left(K_{l} \frac{\partial T_{l}}{\partial x}\right)+g\left(T_{e}-T_{l}\right)$.

Here $S(x, t)$ is the energy source, which describes absorption of laser energy; $x$ is the coordinate toward the target depth (at the surface $x=0) ; C_{e}, C_{l}, K_{e}, K_{l}$ are the heat capacities and thermal conductivities for electrons and lattice respectively; $g$ is the socalled coupling factor, which characterizes the rate of energy exchange between the lattice and the electrons. Note that the term of the lattice thermal conduction in Eq. (2) (first term on the right) was neglected due to a small lattice heat transport on 
sub-nanosecond time scales. The model applied here does not involve considerations of material ablation, which has to be studied with molecular dynamics of hydrodynamics methods [Zhigilei 2009, Povarnitsyn 2007], but allows to evaluate the levels of material heating based on thermodynamic analysis [Shugaev 2010].

Spatial and temporal shapes of the laser pulse intensity I on the target surface can be described by the Gaussian functions as:

$I(r, t)=\frac{2 F(1-R(r, t))}{\tau} \sqrt{\frac{\ln 2}{\pi}} \times$
$\times \exp \left[-4 \ln 2\left(\frac{t-t_{m}}{\tau}\right)^{2}\right] \exp \left[-2\left(\frac{r}{r_{0}}\right)^{2}\right]$

here $F$ is the energy density of laser radiation (usually called fluence); $R$ the reflection coefficient, which can be strongly dependent on material heating, the topic that is addressed in this study; $t_{m}=3 \tau$ is the time between the start of calculations in our model and the peak of laser pulse; $\tau$ is the pulse duration (FWHM that is the full width at half maximum), $r_{0}$ is the radius of the irradiated spot. Then the source term in Eq. (1) is written as

$S(r, x, t)=\alpha(r, x, t) I(r, x, t)$

and the attenuation of laser radiation inside the metal can be described according to the Beer-Lambert law as

$\frac{d I(r, x, t)}{d x}=-\alpha(r, x, t) I(r, x, t)$

where $\alpha$ is the absorption coefficient. Note that this coefficient generally depends on many factors and therefore depends on time and space. Thus, we introduce the laser energy absorption in a more general way (5) than in other studies where an exponential attenuation of the laser light with a constant $\alpha$ value is used.

The initial temperature of electrons and lattice is set to be 300 $K$. The boundary condition on the irradiated surfaces of the metal is the absence of the heat flux through the boundary while, deep inside the metal target, the temperature is considered to be constant and equal to the initial one

$$
\left.\left(\frac{\partial T}{\partial x}\right)\right|_{x=0}=\left.0 \quad T\right|_{x=\infty}=300 \mathrm{~K}
$$

For the thermal capacity, the linear temperature dependence is commonly used while the heat exchange rate between the electron and lattice subsystems is considered constant [Wellershoff 1999]. The thermal conductivity depends on the heat capacity [Lin 2008] and we write

$K_{e}=v_{f}^{2} C_{e} / 3 v_{e} ; C_{e}=A_{e} T_{e} ; g=$ const

Here $v_{F}$ is the Fermi velocity; $v_{e}=A T_{e}^{2}+B T_{\text {l }}$ is the total frequency of electron scattering (in collisions among electrons and with lattice ions). The $A$ and $B$ coefficients were calculated by matching the dielectric function of zinc at room temperature and the melting point with experimentally reported data by applying the Drude model. The proportionality coefficient between the heat capacity of electrons and their temperature in zinc can be evaluated as $A_{e}=\pi^{2} N_{e} k_{B} / 2 T_{F}$ where $N_{e}$ is the density of free electrons and $T_{F}$ is the Fermi temperature [Kittel 1986] (see Table 1).
The Drude model is used to describe the optical properties of zinc with determining the reflection and absorption coefficients via the dielectric function as

$\varepsilon=\varepsilon_{1}+\iota \varepsilon_{2}=\varepsilon_{0}-\frac{\omega_{p}^{2}}{\omega\left(\omega+\iota v_{e}\right)^{\prime}}$

$n=\sqrt{0.5\left(\varepsilon_{1}+\sqrt{\varepsilon_{1}^{2}+\varepsilon_{2}^{2}}\right)}$,

$k=\sqrt{0.5\left(-\varepsilon_{1}+\sqrt{\varepsilon_{1}^{2}+\varepsilon_{2}^{2}}\right)}$

$R=\frac{(n-1)^{2}+k^{2}}{(n+1)^{2}+k^{2}}$

$\alpha=\frac{2 \omega k}{\mathrm{c}}$

Here $\varepsilon$ is the complex dielectric function, which depends on the radiation wavelength $\omega ; \omega_{p}$ is the plasma frequency; $c$ is the speed of light, $n$ and $k$ are refractive and absorption indexes in the media. The $n$ and $k$ values were evaluated from the experimental data [Yarovaya 1974] as 4.88 and 4.65 respectively for the laser wavelength of $800 \mathrm{~nm}$ that corresponds to $~ 65 \%$ of reflectivity.

In practice, the reflectivity of the material samples can differ from the values reported in the handbooks due to different degree of polishing. To address such a possibility in the simulations, we have introduced in the model the effect of surface roughness. We intentionally consider the reflectivity $R=$ 0.57 for zinc at room temperature, approximately $12 \%$ smaller than reported in [Yarovaya 1974], to get insight to the influence of surface roughness.

The rough surfaces can be divided into three groups by the value of the ratio of the mean square surface roughness $\sigma$ to the wavelength $\lambda$ [Wen 2006]. For the group with mirror reflection as normally used in experimental studies of laser-matter interaction (small roughness, $\sigma / \lambda<0.2$ ), the dependence of the reflection coefficient on the roughness is described by the following expression

$R_{r}=R_{p} \exp \left(-\left(\frac{4 \pi \sigma}{\lambda}\right)^{2}\right)$

Here $R_{r}, R_{p}$ are the reflection coefficients of rough and perfectly polished surfaces respectively. Therefore, in our case, reducing the reflectivity by $5.5 \%$ corresponds to the surface roughness $\sigma$ $=23 \mathrm{~nm}$.

\subsection{Material properties}

The investigated material in this work is zinc whose thermophysical parameters are given in Tab. 1:

\begin{tabular}{|l|l|}
\hline Parameters & Value \\
\hline$v_{f},[\mathrm{~m} / \mathrm{s}]$ & $1.83 \times 10^{6}$ \\
\hline$H_{m},\left[\mathrm{~J} / \mathrm{m}^{3}\right]$ & $7.9 \times 10^{8}$ \\
\hline$g,\left[\mathrm{~W} /\left(\mathrm{m}^{3} \mathrm{~K}\right)\right]$ & $2.77 \times 10^{16}$ \\
\hline$C_{l},\left[\mathrm{~J} /\left(\mathrm{m}^{3} \mathrm{~K}\right)\right]$ & 693 \\
\hline$T_{m},[K]$ & 81.9 \\
\hline$A_{e},\left[\mathrm{~J} / \mathrm{m}^{3} \mathrm{~K}^{2}\right]$, & $1.805 \times 10^{6}$ \\
\hline$A,\left[K^{-2} \mathrm{~s}^{-1}\right]$ & $5.5 \times 10^{12}$ \\
\hline$B,\left[K^{-1} \mathrm{~s}^{-1}\right]$ & \\
\hline
\end{tabular}




\section{$\omega_{p},\left[s^{-1}\right]$}

\section{$2.05 \times 10^{16}$}

Table 1. Parameters of zinc used in simulations

Crystalline zinc has a hexagonal close-packed structure similar to that of titanium (Fig. 1). Therefore, it was assumed here that the heat capacity of electrons from a certain temperature should be saturated by analogy with titanium [Lin 2008], which has a similar crystal geometry.

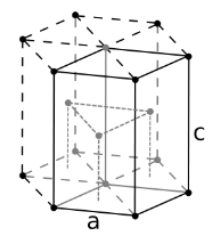

Figure 1. Schematics of the hexagonal close-packed structure of zinc

\subsection{Numerical scheme}

We use an implicit conservative finite-difference scheme with the approximation order $O\left(\Delta x^{2}, \Delta t\right)$ [Godunov 1987]. Such a scheme secures high numerical stability, contrary to the explicit schemes which have a restriction on the time step in order to avoid instable solutions.

In the conservative scheme, the required quantities are the internal energies of electrons $\varepsilon_{e}=\int C_{e} d T_{e}$ and lattice $\varepsilon_{l}=C_{l} T_{l}$ so that the Eqs. (1) and (2) are discretized to the form

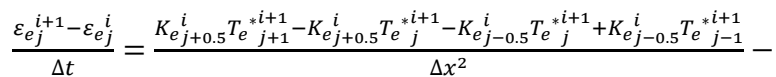

$-g\left(T_{e_{j}}^{i}\right)\left(T_{e_{j}}^{i}-T_{l_{j}}^{i}\right)+S_{j}^{i}$

$\frac{\varepsilon_{l_{j}}^{i+1}-\varepsilon_{l_{j}}^{i}}{\Delta t}=\frac{K_{l}}{C_{l}} \frac{\varepsilon_{l_{j+1}^{i+1}-2 \varepsilon_{l_{j}}^{i+1}+\varepsilon_{l_{j-1}^{i}}^{i+1}}}{\Delta x^{2}}+g\left(T_{e_{j}}^{i}\right)\left(T_{e_{j}}^{i}-T_{l_{j}}^{i}\right)$

$T_{e}{ }_{j}^{i+1}=T_{e_{j}}^{i}+\left(\varepsilon_{e_{j}}^{i+1}-\varepsilon_{e_{j}}^{i}\right) /\left(\frac{\partial \varepsilon_{e}}{\partial T_{e}}\right)_{j}^{i}$

Here and below the subscript $j$ and superscript $i$ refer to the spatial grid points and time layers respectively.

The function $\varepsilon_{l}^{i+1}\left(T_{l}\right)$ takes into account melting processes in the form

$\left\{\begin{array}{c}T_{l}=T_{m}, \text { for } \varepsilon_{l}>C_{l} T_{m} \text { and } \varepsilon_{l}<C_{l} T_{m}+H_{m} \\ T_{l}=\frac{\varepsilon_{l}}{C_{l}}, \text { for } \varepsilon_{l}<C_{l} T_{m} \text { and } \varepsilon_{l}>C_{l} T_{m}+H_{m}\end{array}\right.$

For the value of $\partial \varepsilon_{l} / \partial T_{l}$, we use the following expressions depending on the energy stored in the lattice

$\left\{\begin{array}{l}\frac{\partial \varepsilon_{l}}{\partial T_{l}}=0, \text { for } \varepsilon_{l}>C_{l} T_{m} \text { and } \varepsilon_{l}<C_{l} T_{m}+H_{m} \\ \frac{\partial \varepsilon_{l}}{\partial T_{l}}=C_{l}, \text { for } \varepsilon_{l}<C_{l} T_{m} \text { and } \varepsilon_{l}>C_{l} T_{m}+H_{m}\end{array}\right.$

To solve presented system of equations we used the Tomas algorithm [Godunov 1987]. For this purpose, each equation is written as follows

$A_{j}^{i} X_{j-1}^{i+1}-C_{j}^{i} X_{j}^{i+1}+B_{j}^{i} X_{j+1}^{i+1}=F_{j}^{i}$

The solution is sought in the form

$X_{j-1}^{i+1}=\chi_{j-1}^{i} X_{j}^{i+1}+v_{j-1}^{i}$
$\chi_{j}^{i}=\frac{B_{j}^{i}}{C_{j}^{i}-A_{j}^{i} \chi_{j-1}^{i}} \quad v_{j}^{i}=\frac{A_{j}^{i} v_{j-1}^{i}-F_{j}^{i}}{C_{j}^{i}-A_{j}^{i} \chi_{j-1}^{i}}$

The boundary conditions (6) yield $\chi_{0}^{n}=1, v_{0}^{n}=0$.

To account for the two-dimensionality of the optical problem (for collection of the reflected light over the laser irradiation spot), a uniform grid was introduced along the radius of the irradiation spot $r_{k}$ with a step $d r$. For each value of $r_{k}$ a onedimensional task is solved with energy source given by (3). After calculating the energy $E_{A k}\left(r_{k}\right)$ absorbed at a distance $r_{k}$ from the center of the spot the integral absorbed energy value $E_{A}$ was calculated as follows:

$E_{A}=\frac{\pi}{2} \sum\left(E_{A_{k}}+E_{A_{k+1}}\right) \times\left(r_{k+1}^{2}-r_{k}^{2}\right)$

The laser beam energy integral over the spot was calculated as

$E_{I}=\frac{\pi}{2} \sum\left(S\left(r_{k}\right)+S\left(r_{k+1}\right)\right) \times\left(r_{k+1}^{2}-r_{k}^{2}\right)$

The final value of the integral reflectivity can be found as

$R=1-E_{A} / E_{I}$

We chose the irradiation spot radius $r_{s}$ and the numerical grid steps along the sample surface $d r$ so that increasing the radius or decreasing the spatial step does not noticeably affect the results of the energy integrations.

\section{RESULTS}

All the calculations given below are performed for the following conditions of laser irradiation used in many experiments, in particular in [Vorobyev 2009, Kirkwood 2009, Vorobyev 2011]: wavelength $800 \mathrm{~nm}$, pulse duration $66 \mathrm{fs}$, average radius of the irradiated spot $50 \mu \mathrm{m}$. The laser fluence ranged from $0.01 \mathrm{~J} / \mathrm{cm}^{2}$ (significantly below the melting thresholds of zinc) to $20 \mathrm{~J} / \mathrm{cm}^{2}$ (strong ablation regime).

In order to determine the optimal step of the calculated grid, methodological simulations were carried out. The absorption depth in zinc under the normal conditions is app. $13.7 \mathrm{~nm}$ for 800 $\mathrm{nm}$ wavelength and, hence, the spatial step has to be smaller than the absorption depth. It was found that the spatial step $\Delta x$ $=1 \mathrm{~nm}$ is satisfactory and, with its further decrease, the simulation results for all parameters change negligibly. Thus, all simulations were performed with $\Delta x=1 \mathrm{~nm}$. The time step was set as follows:

$$
\left\{\begin{array}{c}
\Delta t=1 \mathrm{fs}, \text { for } t<1000 \mathrm{fs} \\
\Delta t=10 \mathrm{fs}, \text { for } t>1000 \mathrm{fs}
\end{array}\right.
$$

Figure 2 shows the dependence of the integral reflection coefficient on the radius of the integration at the radial grid step $\Delta r=7.5 \mu \mathrm{m}$. The calculations used the integration area of the radiation spot radius $r_{s}=100 \mu \mathrm{m}$ provide a sufficient accuracy. Similarly, for the case of simulations for the radiation spot radius $r_{s}=100 \mu \mathrm{m}$, the satisfactory accuracy with the radial step $\Delta r=$ $7.5 \mu \mathrm{m}$ was confirmed. 


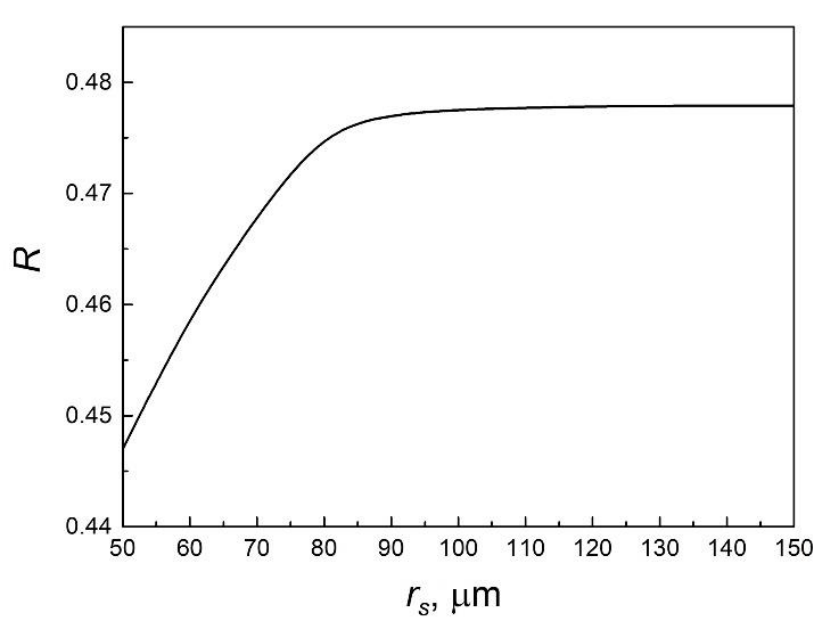

Figure 2. Integral reflectivity dependence on the spot radius involved into integration at a fixed radial grid step of $7.5 \mu \mathrm{m}$.

The dynamics of the electron and lattice temperatures on the irradiated surface as a function of time is shown in Fig. 3 for the laser fluence $F=1 \mathrm{~J} / \mathrm{cm}^{2}$. It demonstrates that, during the action of a femtosecond laser pulse, the lattice stays cold while the electrons temperature increases sharply up to about the Fermi temperature even at such a moderate fluence. Due to a relatively low thermal conductivity of electrons in zinc, the electron temperature is decreasing mostly because of energy transfer to the lattice. As a result, the lattice is overheated at a surface layer up to tens of thousands degrees and should be transformed into the state of gas plasma before the completion of the electron-lattice relaxation, as analysed in [Bulgakova 2002b].

Although the simulations do not account for material ablation, the calculated temperature evolution can give a valuable information on a possible scenario of material decomposition. The thermodynamic critical temperature of zinc can be evaluated as $3190 \mathrm{~K}$ [Fortov 1985]. In the surface layer, such level of the lattice temperature can be reached already at $\sim 3 p s$ after the laser pulse action and material can start to swiftly decay via phase explosion into the mixture of atomic phase and droplets [Zhigilei 2009]. At this time moment, the temperature of electrons is still much higher than the lattice temperature. This implies that the electron-lattice thermalization will continue inside the droplets. As a result, the droplets will vaporize and, from the energy balance consideration, one may expect complete atomization of the expanding vapour-droplet phase and plausibly partial ionization. Such scenario can be verified by molecular dynamic simulations [Zhigilei 2009].

The temperature profiles for the electron and lattice subsystems are presented in Figs. 4 and 5 for the time moment immediately after the laser pulse action and for 90 ps when the electronlattice themalization has been reached. From these Figures, it is seen that the absorbed energy is not penetrate deep to the sample. Instead, the layer of the order of $\sim 100 \mathrm{~nm}$ is strongly overheated (Fig. 5) that must cause a swift ablation of this layer, as described above, in the form of phase explosion followed by evaporation of the droplets and partial ionization.

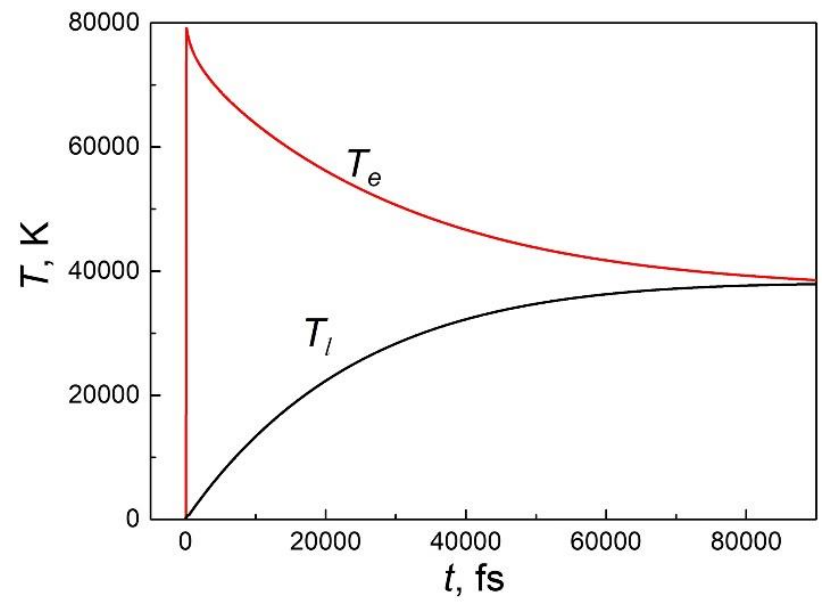

Figure 3. Typical temporal evolution of the electron and lattice temperatures on the surface of the irradiated zinc at $F=1 \mathrm{~J} / \mathrm{cm}^{2}$

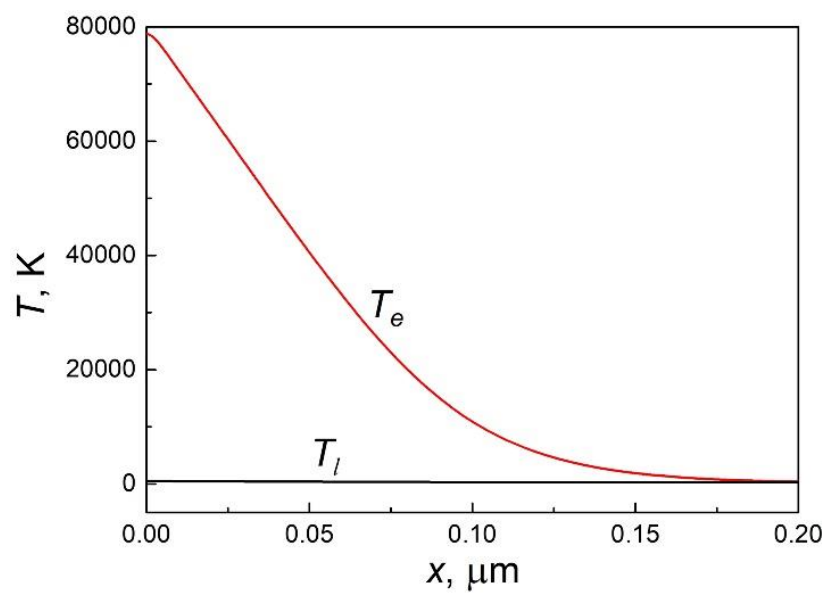

Figure 4. Typical temperature distribution just after the end of laser pulse action at $F=1 \mathrm{~J} / \mathrm{cm}^{2}$

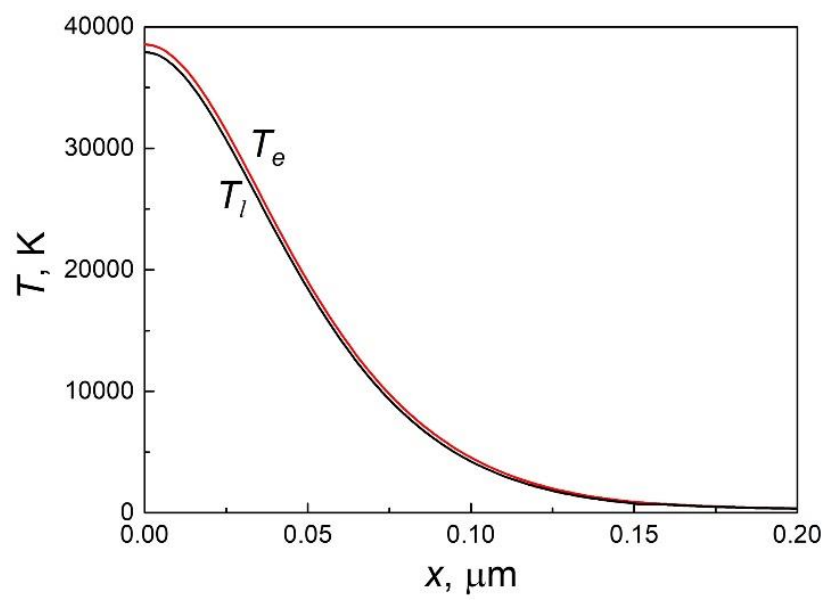

Figure 5. The same as in Fig. 5 for the time moment $t=90 \mathrm{ps}$

Since the model predicts that the electron temperature in zinc can reach very high values even in the case of moderate laser fluences, the heat capacity of electrons, being implemented as a linear function of $T_{e}$, can grow to physically ungrounded values (above the values of an ideal electron gas). Hence, the heat capacity must saturate at some temperature with a maximum inherent for the behavior of an ideal plasma. We note that the electron heat capacities of most metals calculated based of the density of states theory [Lin 2008] show a tendency of saturation at high temperatures and their values do not exceed $1.5 k_{B}$ where $k_{B}$ is the Boltzmann constant. Here we assume for simplicity that the electron heat capacity increases linearly only to a certain 
temperature (5000 K, $15000 \mathrm{~K}$ and $35000 \mathrm{~K})$ and then it saturates at this maximum level with further temperature increase.

Figure 6 shows the comparison of the integral reflectivity dependences on laser fluence calculated with the infinite linear increase of the electron heat capacity and for the saturation of the heat capacity at the three mentioned levels. It is seen the choice of the heat capacity behaviour with the temperature dramatically influences the optical response of zinc irradiated by ultrashort laser pulses.

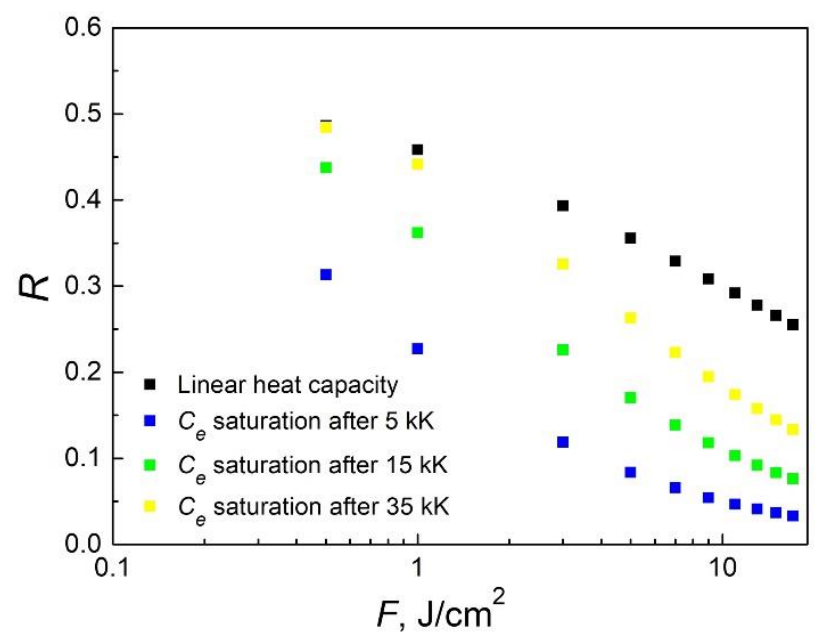

Figure 6. The dependences of integral reflectivity on laser fluence predicted numerically with different values of the electron heat capacity saturation.

An important conclusion can be made that the correct determination of the thermophysical parameters of the free electron gas, in particular the heat capacity, is extremely important to describe adequately experimental data on the metal reflectivity. The simulations predict a strong dependence of the optical response of metals on their thermophysical properties, in agreement with the tendencies observed experimentally [Kirkwood 2008, Vorobyev 2011]. We can speculate that, by comparing the numerical results of the present model with measurements of fluence-dependent reflectivity, it could be possible to gain an insight into behaviour of the electron heat capacity as this parameter appears to have a strongest impact on the dynamics of optical properties upon excitation with ultrashort laser pulses.

\section{CONCLUSIONS}

In this paper, a comprehensive model has been developed targeted on advanced comparisons of the modelling results with experimental data. The model is based on two-temperature simulations of the laser excitation of material and involves a rigorous modelling of optical response of metallic material that includes integration of the reflected energy over the laser irradiation spot. The numerical code was developed using the conservative scheme and its stability and accuracy have been verified. A series of calculations have been performed for zinc as an example. It was shown that zinc can be heated to extremely high temperatures even at moderate laser fluences. Finally, the predictions on reflectivity behaviour of zinc have been made that call for further studies involving reflectivity measurements.

\section{ACKNOWLEDGMENTS}

This work was supported by the Ministry of Education, Youth and Sports of the Czech Republic (Programmes NPU I Project No.
LO1602, and the Large Research Infrastructure Project No. LM2015086). S.A.L. and A.V.B. also acknowledge financial support from the Russian Foundation for Basic Research (project No. 19-38-90203).

\section{REFERENCES}

[Anisimov 1974] Anisimov, S.I., et al. Electron emission from metal surfaces exposed to ultrashort laser pulses, Sov. Phys. JETP, 1974, Vol.39, pp 375-377.

[Ashitkov 2016] Ashitkov, S.I., et al. Ablation of gold irradiated by femtosecond laser pulse: Experiment and modelling, J. Phys.: Conf. Ser., 2016, Vol. 774, Article 012097.

[Bulgakova 2002a] Bulgakova, N.M. and Bourakov, I.M. Phase explosion under ultrashort laser ablation: Modeling with analysis of metastable state of melt, Appl. Surf. Sci., 2002, Vol.197-198, pp 41-44.

[Bulgakova 2002b] Bulgakova, N.M., et al. Pulsed laser ablation of solids and critical phenomena, Appl. Surf. Sci., 2002, Vol.197198, pp 96-99.

[Chen 2013] Chen, Z., et al. Evolution of ac conductivity in nonequilibrium warm dense gold. Phys. Rev. Lett., 2013, Vol.110, Article 135001.

[Dostovalov 2019] Dostovalov, A.V., et al. LIPSS on thin metallic films: New insights from multiplicity of laser-excited electromagnetic modes and efficiency of metal oxidation, Appl. Surf. Sci., 2019, Vol.491, pp 650-658.

[Eliezer 2004] Eliezer, S., et al. Synthesis of nanoparticles with femtosecond laser pulses. Phys. Rev. B, 2004, Vol. 69, Article 144119.

[Fang 2017] Fang, R., et al. Direct visualization of the complete evolution of femtosecond laser-induced surface structural dynamics of metals, Light. Sci. Appl., 2017, Vol. 6, Article e16256.

[Fortov 1975] Fortov, V.E., et al. Evaluation of the parameters of the critical point. High Temp. (USSR), 1975, Vol. 13, pp 984-992.

[Godunov 1987] Godunov, S. K. and Ryabenkii, V. S. Difference schemes, Elsevier Science Publishers B.V., 1987, ISBN-13: 0444702334.

[Kirkwood 2009] Kirkwood, S.E., et al. Experimental and theoretical study of absorption of femtosecond laser pulses in interaction with solid copper targets. Phys. Rev. B, 2009, Vol.79, Article 144120.

[Kittel 1986] Kittel, C. Introduction to Solid State Physics, 6th ed., Wiley, New York, 1986. ISBN-10: 0471874744 / ISBN-13: 9780471874744.

[Klini 2008] Klini, A., et al., Laser induced forward transfer of metals by temporally shaped femtosecond laser pulses, Opt. express, 2008, Vol. 16, pp 11300-11309.

[Lin 2008] Lin, Z., et al. Electron-phonon coupling and electron heat capacity of metals under conditions of strong electronphonon nonequilibrium, Phys. Rev. B, 2008, Vol.77, Article 075133. 
[Mailis 1999] Mailis, S., et al. Etching and printing of diffractive optical microstructures by a femtosecond excimer laser. Appl. Optics, 1999, Vol.38, No.11, pp 2301-2308.

[Ren 2011] Ren Y. et al. Ultrashort laser pulse energy deposition in metal films with phase changes, Appl. Phys. Lett., 2011, Vol. 98, Article 191105

[Povarnitsyn 2007] Povarnitsyn, M.E., et al. Multi-material twotemperature model for simulation of ultra-short laser ablation, Appl. Surf. Sci., 2007, Vol. 253, pp 6343-6346.

[Shugaev 2010] Shugaev, M.V. and Bulgakova, N.M. Thermodynamic and stress analysis of laser-induced forward transfer of metals, Appl. Phys. A, 2010, Vol. 101, pp 103-109.

[Vorobyev 2009] Vorobyev, A.Y. and Guo, C. Metal pumps liquid uphill. Appl. Phys. Lett., 2009, Vol.94, Article 224102.

[Vorobyev 2011] Vorobyev, A.Y. and Guo, C. Reflection of femtosecond laser light in multipulse ablation of metals. J. Appl. Phys., 2011, Vol.110, Article 043102.

\section{CONTACTS:}

M.S. Sergey A. Lizunov

Prof. Vladimir P. Zhukov, Dr.Sc.

Prof. Alexander V. Bulgakov, Dr.Sc.

Prof. Nadezhda M. Bulgakova, Dr.Sc.

HiLASE Centre, Institute of Physics of the Czech Academy of Sciences

Za Radnicí 828, Dolní Břežany 25241 , Czech Republic

+420-314007709; lizunov@fzu.cz; https://www.hilase.cz/s
[Vorobyev 2015] Vorobyev, A.Y. and Guo, C. Multifunctional surfaces produced by femtosecond laser pulses. J. Appl. Phys., 2015, Vol.117, Article 033103.

[Wellershoff 1999] Wellershoff S., et al. The role of electronphonon coupling in femtosecond laser damage of metals," Appl. Phys. A, 1999, vol.69, pp S99-S107.

[Wen 2006] Wen, C.-D. and Mudawar, I. Modeling the effects of surface roughness on the emissivity of aluminum alloys. Int. J. Heat Mass Trans., 2006, Vol.49, pp 4279-4289.

[Yarovaya 1974] Yarovaya R.G. et al. Temperature dependence of the optical properties and the energy spectrum of zinc, Sov. Phys. - JETP, 1974, Vol. 38, No. 2, pp 331-334.

[Zhigilei 2009] Zhigilei L.V., et al. Atomistic modeling of short pulse laser ablation of metals: Connections between melting, spallation, and phase explosion, J. Phys. Chem. C, 2009, Vol. 113, pp. 11892-11906. 